\title{
Gastric cancer in Singapore
}

\author{
Melvin Look ${ }^{1}$, Fei GaO ${ }^{2}$, Cheng Hock Low ${ }^{1}$, and Raj Nambiar ${ }^{1}$ \\ ${ }^{1}$ Department of Surgery, Tan Tock Seng Hospital, 11 Jalan Tan Tock Seng, Singapore 308433, Republic of Singapore \\ ${ }^{2}$ Division of Clinical Trials and Epidemiological Sciences, National Cancer Centre, Singapore
}

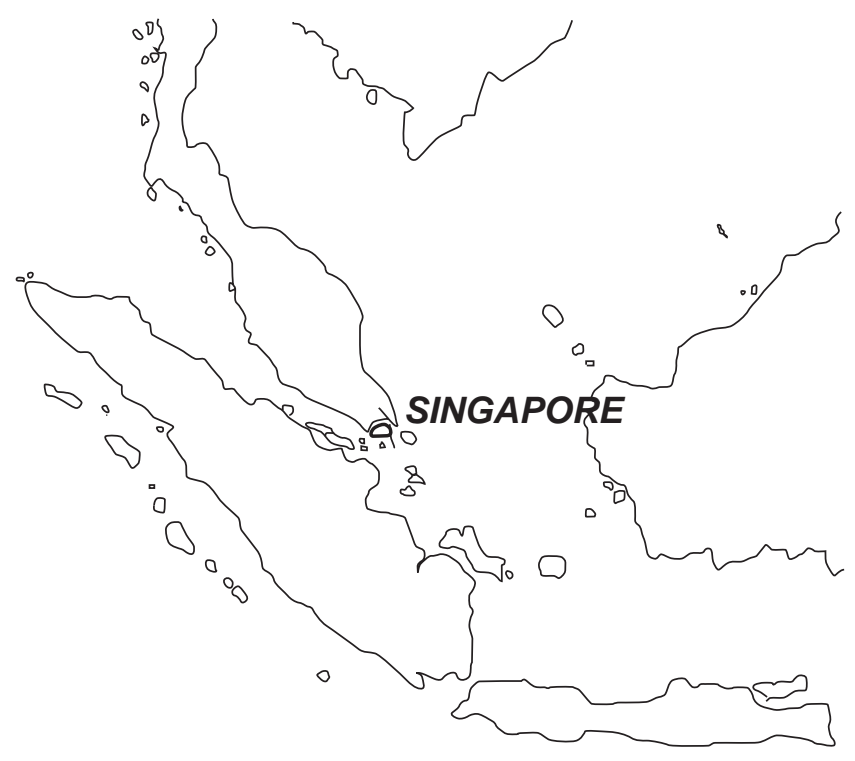

Key words Gastric cancer · Epidemiology $\cdot$ Incidence

\section{Introduction}

Gastric cancer is one of the commonest malignancies worldwide and is one that displays interesting differences between Western and Eastern countries. Despite declining temporal trends in incidence, there is an inexplicable and almost epidemic rise in the number of cardia cancers in Western patients. Epidemiological

Offprint requests to: $\mathrm{M}$. Look

Received: August 1, 2001 / Accepted: October 15, 2001 reports of individual countries may help to further our understanding of this disease.

\section{Population demographics}

Singapore is a small island republic in South East Asia with a population of approximately 3.5 million people, of which residents make up nearly $90 \%$. Of these, $77.7 \%$ are Chinese; $14.1 \%$, Malays; $7.1 \%$, Indians; and $1.8 \%$, other ethnic groups. The overall ratio of males to females is $1026: 1000$, with a slightly higher male preponderance in Indians.

Since the gaining of political independence in 1965 , there has been rapid economic and social development. Singapore is now a well-developed city-state with a high standard of medical care that is affordable and readily accessible. More than 20 public and private hospitals provide in excess of 10500 hospital beds (2.9 beds per 1000 population). The population-to-doctor ratio has improved steadily over the past 30 years, and is 760:1 at present.

\section{Cancer statistics in Singapore}

Cancer has risen in importance over the years as a cause of death in Singapore, and it is now the leading cause of death. The crude annual cancer death rate is now 111.9 per 100000 population, and cancer accounts for $25.6 \%$ of all causes of death, compared with $14.8 \%$ three decades ago [1].

Lung cancer is the commonest cancer in males, followed by colorectal, gastric, liver, and nasopharyngeal cancers. In females, the commonest cancers are breast, colorectal, lung, cervical, and gastric. The crude rate (per 100000 population per year) and age-standardized rate (ASR, per 100000 population per year) for gastric 
cancer are 18.6 and 21.0, respectively, in males, and 12.3 and 11.3 , respectively, in females.

\section{Ethnic group and age patterns of gastric cancer}

There are distinct differences in the incidence of gastric cancer between the various ethnic groups in Singapore. The incidence is highest in Chinese males (ASR, 25.7), in whom it is the third commonest cancer, and considerably lower in Indian and Malay males (ASR, 8.4, and 6.6 , respectively). In females, the incidence is correspondingly lower (ASR, 12.6, 6.3, and 4.0 in Chinese, Indians and Malays, respectively).

The age-adjusted relative risk compared with Chinese as a reference group is, therefore, 0.34 (95\% confidence interval [CI], 0.26 to 0.44 ) for Indian males and 0.28 (95\% CI, 0.22 to 0.37 ) for Malay males. Likewise, the relative risk is 0.52 ( $95 \% \mathrm{CI}, 0.34$ to 0.78 ) for Indian females and 0.34 (95\% CI, 0.24 to 0.49$)$ for Malay females.

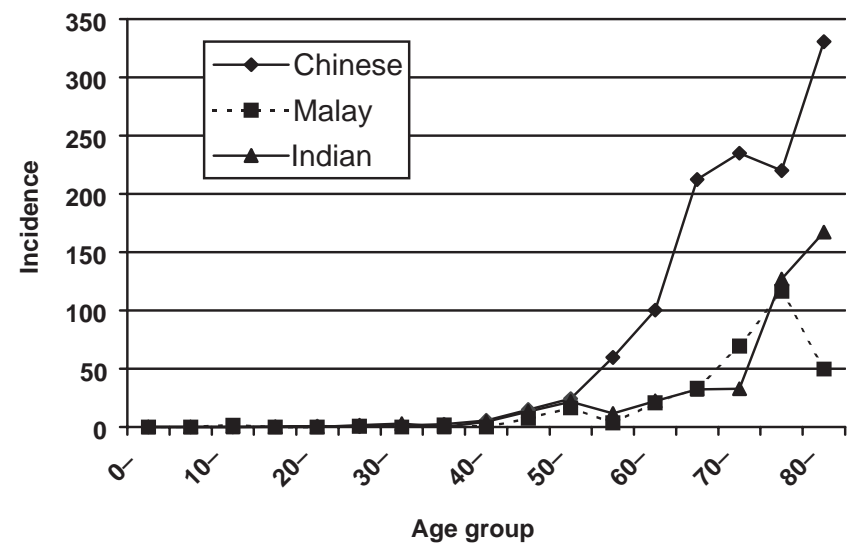

Fig. 1. Incidence (per 100000 per year) of gastric cancer in males by age group for the different ethnic groups

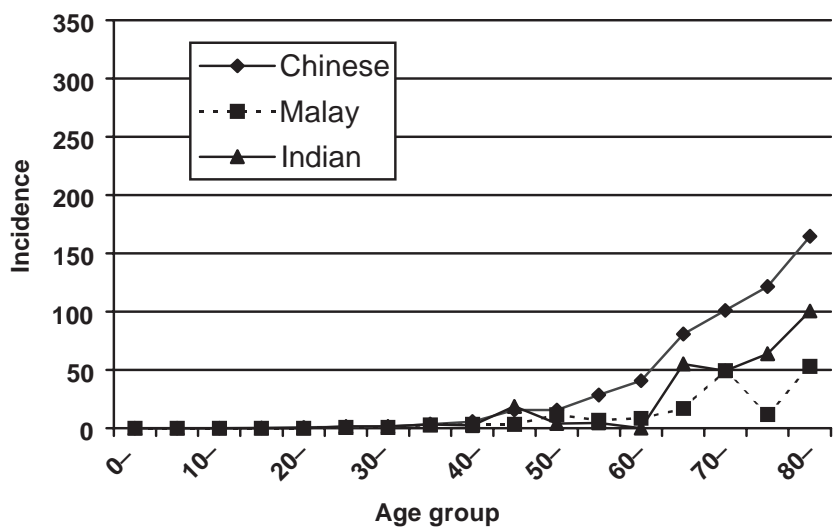

Fig. 2. Incidence (per 100000 per year) of gastric cancer in females by age group for the different ethnic groups
The majority of Chinese in Singapore are first- or second-generation descendants of migrants from various regions in China. Even in the Chinese, this malignancy seems to have a predilection for certain dialect groups. Hokkiens and Teochews have a higher risk than Cantonese, Hakka, Hainanese, and others. Foreignborn Chinese have higher risks than the local-born. In contrast, the converse is true for colorectal cancers in Chinese in Singapore. International comparisons of ASRs reveal that the incidence of gastric cancer in Singapore Chinese is lower than that in Chinese in Shanghai, but higher than that in Chinese in Hong Kong, Hawaii, and Los Angeles [1].

Gastric cancer in Singapore is rare in children and young adults. Its incidence starts to rise in middle-aged adults (age, 35 to 64 years) in which population it accounts for $7.6 \%$ and $3.9 \%$ of all cancers in males and females, respectively. It is even more common in the elderly (age, 65 years and above), accounting for $10.5 \%$ and $9.2 \%$ of all cancers in this age group for males and females, respectively. The incidence peaks in males over 80 and, in this group, it approaches 300 per 100000 per year. The incidence by age group in the three major ethnic groups is shown in Figs. 1 and 2 for males and females, respectively.

\section{Trends in gastric cancer incidence}

Gastric cancer was the cancer of highest incidence in Singapore in the 1960s, but, along with esophageal and liver cancers, it showed one of the steepest declines in incidence among all cancers over the past three decades (see Fig. 3). The ASR has fallen steadily to its present

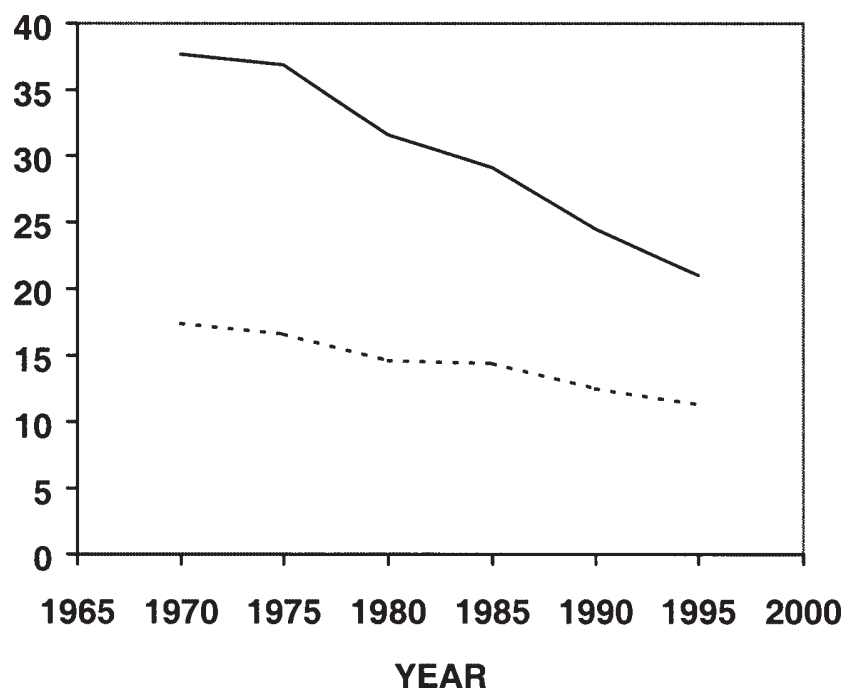

Fig. 3. Age-standardized incidence of gastric cancer by sex, 1968-1997. Continuous line, Males; dashed line, females 
levels, from a high of 37.7 in males and 17.4 in females, for the 5-year period of 1968 to 1972.

The average annual percent change in ASRs in the period 1968 to 1997 is minus $2.18 \%$ in males and minus $1.63 \%$ in females. In comparison, cancers that have risen in incidence in the corresponding period include lymphomas and prostate, breast, and colorectal cancers.

\section{Risk factors}

The reasons for the high incidence of gastric cancer in Singapore are not completely clear.

Familial factors and genetic susceptibility probably play a strong role in the differences seen between ethnic and dialect groups.

The falling rates of gastric cancer in Singapore over the years reflect a similar trend worldwide, and may be associated with the availability of domestic refrigeration and the dietary changes that occurred during the period of rapid socioeconomic transformation after World War II. The intake of salted, smoked, pickled, and preserved foods decreased, while fresh fruit, vegetables, and sources of protective micronutrients, such as ascorbic acid and beta-carotene, have become readily available.

Helicobacter pylori infection has been accepted as a class I carcinogen for the development of gastric cancer, although a definite causal model does not exist. The prevalence of $H$. pylori infection in the Singapore adult population shows a distinct ethnic pattern. It is present in $70 \%$ of Indians, $43 \%$ of Chinese, and $36 \%$ of Malays [2]. The ethnic differences in gastric cancer frequency, therefore, cannot be explained by $H$. pylori infection alone, and may possibly be caused by host-bacterial interaction factors [3].

\section{Clinicopathological factors}

Adenocarcinomas account for $92.7 \%$ of all gastric tumors in Singapore. Other histological types include gastric lymphomas $(4.0 \%)$, stromal tumors $(2.2 \%)$, neuroendocrine tumors $(0.3 \%)$, and other rare types, such as squamous cell carcinomas and malignant melanomas.

Distal gastric cancers tend to outnumber proximal tumors in our local population. Malignancies of the cardia and proximal stomach account for between $15 \%$ and $27 \%$ of all cases [4]. The site distribution has remained consistent for the past three decades, and is quite unlike the near epidemic rise in cancers of the esophagogastric junction seen in Western countries during the same time period. In addition, adenocarcinomas of the esophagus are rare in Singapore, and this may be because gastroesophageal reflux disease is uncommon in Asians.
The majority of patients with gastric cancer in Singapore present when the disease is at an advanced stage. Three-quarters of all patients have stage III or IV disease at the time of diagnosis, and one-fifth will have metastatic disease. Nodal dissemination is present in $70 \%$ of patients [4]. On the other hand, fewer than $10 \%$ of gastric cancers in Singapore are early gastric cancers (EGCs) [5]. Nearly all patients with EGCs in Singapore present with symptoms, as there is no screening program for an asymptomatic population in this country [6]. Nevertheless, endoscopy is readily available and is the main mode of investigation for patients with gastrointestinal complaints.

\section{Treatment and results}

Surgery is the mainstay of treatment for gastric cancer. The curative resection rate has improved over the years, possibly because of better preoperative staging, and is currently about $33 \%$ [4]. The overall prognosis has remained poor, with post-resection 2-year survival rates of around $40 \%$ in the past decade. Such surgery has traditionally been performed by general surgeons. In the past few years, however, greater subspecialization has seen a move towards more radical operations, conducted by upper gastrointestinal surgeons who perform systematic lymphadenectomy, following the Japanese technique. Adjuvant and neoadjuvant chemotherapy and radiotherapy are also increasingly popular. The outcome of such an approach seems promising, but long-term results are awaited.

\section{Conclusion}

The Ministry of Health has set a national target for reducing cancer mortality by $10 \%$ over the next 20 years. Although the incidence of gastric cancer in Singapore is falling, much remains to be done to reduce the mortality from this disease. To achieve this, a strategy of population education to promote healthy lifestyles and to encourage early presentation for diagnosis of symptoms has been set in place. Improved cancer care will be delivered through two designated comprehensive cancer centers. These facilities will aim to combine the best of a multidisciplinary, scientific approach to cancer treatment, with a holistic outlook, to provide the best care possible for gastric cancer patients in Singapore.

Acknowledgments We thank Professor K. Shanmugaratnam and the Singapore Cancer Registry for their help in preparing this manuscript. 


\section{References}

1. Chia KS, Seow A, Lee HP, Shanmugaratnam K. Cancer incidence in Singapore 1993-1997. Singapore Cancer Registry, report No. 5. Singapore; 2000.

2. Kang JY, Yeoh KG, Ho KY, Guan R, Lim TP, Quak SH, et al. Racial differences in Helicobacter pylori seroprevalence in Singapore: correlation with differences in peptic ulcer frequency. $J$ Gastroenterol Hepatol 1997;12:655-9.

3. Yeoh KG. Helicobacter pylori infection and its treatment in Singapore. Singapore Med J 2000;41;175-7.
4. Koong HN, Chan HS, Nambiar R, Soo KC, Ho J, Ng HS. Changes in the outcome of gastric cancer in Singapore over the last three decades. J Gastroenterol Hepatol 1996;11(Suppl 2):A51-A77.

5. So JBY, Samarasinghe K, Raju GC, Ti TK. Clinical and pathological features of early gastric cancer in Singapore. Ann Coll HK 2001;5:10-3.

6. Look M, Tan YY, Vijayan A, Teh E, Low CH. Management delays for early gastric cancer in a country without mass screening. Hepatogastroenterology (in press) 